Service social

\title{
Un savoir à notre image? Critiques féministes des disciplines, sous la direction de Roberta Mura, Montréal, Les éditions Adage Inc., coll. EF, 1991, 296 pages.
}

\section{Céline Bédard}

Volume 41, numéro 1, 1992

L’avenir des services ou services d'avenir

URI : https://id.erudit.org/iderudit/706564ar

DOI : https://doi.org/10.7202/706564ar

Aller au sommaire du numéro

Éditeur(s)

École de service social de l'Université Laval

ISSN

1708-1734 (numérique)

Découvrir la revue

Citer ce compte rendu

Bédard, C. (1992). Compte rendu de [Un savoir à notre image? Critiques féministes des disciplines, sous la direction de Roberta Mura, Montréal, Les éditions Adage Inc., coll. EF, 1991, 296 pages.] Service social, 41(1), 166-168. https://doi.org/10.7202/706564ar d'utilisation que vous pouvez consulter en ligne.

https://apropos.erudit.org/fr/usagers/politique-dutilisation/ 
L'œuvre commune à laquelle j'ai accepté de collaborer - c'est-à-dire la production d'un manuel d'introduction à l'organisation communautaire vise notamment à améliorer la qualité de la formation au baccalauréat en service social. Dans un chapitre sur l'action conscientisante, j'ai présenté les sources, le processus type, les apports et les limites de ce type d'intervention. En écrivant ce texte, je n'ai voulu être ni le chantre ni le détracteur d'une pratique significative en organisation communautaire; mon article témoigne de l'importance qu'il faut accorder à cette école d'intervention mais aussi des limites qu'elle comporte. Une attitude complaisante $m^{\prime}$ aurait peut-être valu de la part du recenseur des félicitations ou un silence complice. J'ai choisi la voie de la rigueur, ce qui n'est pas le cas de la recension de mon collègue $G$. Doré.

Le droit à la critique s'accompagne du devoir de l'accepter lorsqu'elle nous est lancée. Dans un cas comme dans l'autre, les règles scientifiques doivent être respectées. Je constate que la critique de mon texte ne satisfait pas ces critères : elle n'est appuyée sur aucune preuve; elle condamne sans démontrer. Dommage, car l'auteur évite ainsi un débat qui aurait pu enrichir l'approche conscientisante et nourrir l'organisation communautaire. Cependant, c'est un mal pour un bien que ce débat n'ait pas eu lieu, au vu du sort qu'il réserve aux deux dernières phrases de mon texte; qu'on en juge :

"Elle contribue ainsi à créer une image d'un noyau fermé sur luimême, ancré dans sa doctrine, étanche à toutes influences externes. L'analogie avec une certaine façon de pratiquer le catholicisme et avec le marxisme-léninisme est facile à faire. "

Les mots soulignés sont ceux que Gérald Doré a négligé de présenter aux lecteurs de sa recension; et pourtant que de différence entre les deux phrases. Tout commentaire additionnel est superflu. Le lecteur comprendra que je préfère discuter avec celles et ceux qui ne sont pas en possession tranquille de la vérité.

Yves Hurtubise

\section{Un savoir à notre image ? - Critiques féministes des disci-} plines, sous la direction de Roberta Mura, Montréal, Les éditions Adage inc., coll. EF, 1991, 296 pages.

Cet ouvrage, écrit par une vingtaine d'auteures, rassemble les textes présentés au séminaire Critiques féministes du savoir, organisé par le Groupe de recherche multidisciplinaire féministe de l'Université Laval (GREMF) en 1986-1987 et en 1987-1988.

Le but de ce séminaire était d'examiner l'apport d'une perspective féministe à la vision de certains champs de savoir, tels ceux de : l'histoire, la littérature, la musique, les sciences pures, la biologie, l'économie, la science politique, la psychologie, la psychothérapie, la psychanalyse, le développement moral, l'architecture, le service social, le droit et la théologie (d'autres disciplines, également représentées au séminaire, n'ont pu faire l'objet d'un compte rendu dans cet ouvrage).

L'objectif n'était pas de déplorer l'absence des femmes dans les lieux du savoir, mais de découvrir les marques laissées par l'idéologie patriar- 
cale, par les préjugés culturels ou sexistes sur ce même savoir, et de tenter de voir comment une critique féministe pouvait contribuer à produire un savoir non sexiste.

Quinze disciplines ont ainsi été étudiées sous différents aspects, à partir de plusieurs visions et avec des conclusions et des résultats différents, car toutes ne répondent pas également à une même analyse féministe, l'effet de l'androcentrisme étant plus ou moins important selon les disciplines. Malgré cela, un même consensus s'en dégage : les femmes ne se sont pas reconnues dans l'image que leur renvoie le savoir traditionnel; cette image est déformée, négligée, infériorisée et parfois même inventée.

Que l'on examine l'histoire, la science politique ou le développement moral, on constate que les femmes en ont été le plus souvent bannies; étant cataloguées sous le concept universel d'humain et les observations faites à partir d'exemples uniquement masculins, elles s'en trouvaient déjà exclues. En économie, on a ignoré les activités économiques des femmes; l'architecture au féminin aurait voulu s'intéresser aux besoins quotidiens de la femme plutôt qu'au style du bâtiment. La psychologie a rendu la femme responsable des problèmes de ses enfants, alors qu'en théologie, c'est Ève qui porte tous les péchés de l'humanité. Les sciences pures, n'ayant pas le féminin comme objet d'études, semblent échapper à la condamnation; mais encore faudrait-il regarder les méthodes utilisées, le langage tenu, I'utilisation faite des résultats de leurs recherches pour l'affirmer.

Ce schéma, simplifié ici à outrance, peut se répéter pour chacune des disciplines, et n'en montre pas moins un savoir imbu de préjugés sexistes et de misogynie qui contribuent à alimenter la dévalorisation des femmes.

Comment cela peut-il être encore le cas, quand les femmes ont massivement envahi l'université - elles représentent maintenant plus de 50 pour 100 de la clientèle étudiante - et conquis les disciplines traditionnellement masculines?

Est-ce à dire que la démarche est vouée à l'échec ? Ou alors, un savoir uniquement féminin serait-il la solution ? Autrement, un savoir neutre serait-il possible? II n'est pas aisé de répondre à ces questions et de trouver la juste solution, sans envisager d'abord une transformation radicale et une redéfinition des disciplines. Les femmes, pour leur part, désirent la reconnaissance de leurs différences; elles demandent qu'une place soit faite aux valeurs humaines d'égalité, d'entraide, de solidarité; qu'un lien soit établi entre pensée et sentiment, entre esprit et matière, entre corps et âme, entre théorie et pratique.

Même s'il est évident que la lutte des femmes depuis une vingtaine d'années a fait avancer la discussion, il reste encore beaucoup à faire dans les tours d'ivoire que sont nos universités. Voilà pourquoi une publication telle que celle qui est offerte à notre réflexion demeure des plus utiles pour élargir le cadre du débat et favoriser les échanges d'opinions.

Parce que le contenu intéresse au premier chef les universitaires, cet ouvrage devrait être lu par tous les étudiants et toutes les étudiantes, tout au moins les articles concernant leur discipline propre. II devrait également faire partie de la bibliothèque de chacun des professeurs et chacune des professeures. À cause de l'hermétisme de certains articles, je crains cepen- 
dant qu'il ne rebute un lecteur non averti. L'introduction ainsi que la conclusion de Mme Roberta Mura contribuent pour beaucoup à maintenir la continuité du sujet, et cela facilitera la lecture de tout l'ouvrage.

Enfin, la qualité d'impression et de présentation est excellente; les différents textes sont aérés et se consultent aisément. Le volume a le grand mérite de fournir une référence bibliographique des plus récentes.

\author{
Céline Bédard \\ École de service social \\ Université Laval
}

\title{
Programme Coup de Main, "Guide d'animation d'ateliers de soutien pour aidantes naturelles ». Coordination : Danielle MALTAIS, DSC - Hôpital général de Montréal, Serge NAULT, DSC - Centre hospitalier de Verdun, 1991, 254 pages.
}

Ce cahier à feuilles mobiles est un guide d'animation. Il s'adresse aux animatrices d'ateliers qui offrent aux aidantes naturelles responsables d'un proche en perte d'autonomie un service de soutien dans leur travail bénévole. Le cahier propose seize thèmes pouvant servir d'instruments d'animation pour autant de rencontres de groupe correspondant à des besoins préalablement déterminés.

L'introduction définit d'abord la clientèle visée, soit les aidantes naturelles. On y prend en considération plus d'une vingtaine de recherches et d'études effectuées à ce sujet (Paquette, 1988; Lepage, 1989; Gonyea, 1989; Haley, 1989; Toseland Rossiter, Labrecque, 1989; Maltais, 1990, etc.). Fait à noter, les limites propres à l'utilisation des groupes de soutien pour aidantes naturelles y sont précisées avec une grande netteté. Ainsi l'étude de Garant et Bolduc (1990) souligne que " certains chercheur-e-s ont constaté que la participation à des ateliers de soutien peut apporter des effets négatifs sur certaines aidantes ». Devant ces limites, on présente le guide comme n'étant qu'un instrument parmi d'autres sur ies services à offrir aux aidantes. Cette présentation est un incitatif à la prudence. L'utilisation de ce guide doit en effet s'insérer dans un processus d'ensemble où il ne sera finalement qu'un élément complémentaire à d'autres mesures d'aide ou de soutien.

Le mode d'utilisation du guide est, dès le début, explicité sous divers angles : l'objectif général des ateliers où il doit être utilisé, le modèle d'intervention dont il s'accommode le mieux, la démarche andragogique, les ateliers et les thèmes proposés, puis l'organisation, le déroulement et l'évaluation de ces ateliers et, finalement, ce que le guide appelle " l'aprèsatelier».

Dans cette section concernant le mode d'utilisation, nous retrouvons les principes directeurs, les idéologies sous-jacentes, de même que les détails de l'organisation technique et les qualités attendues des animatrices. En procédant ainsi, les auteurs respectent les éléments relevant de la phase pré-groupe, soit la recherche d'informations préliminaires, la clari- 